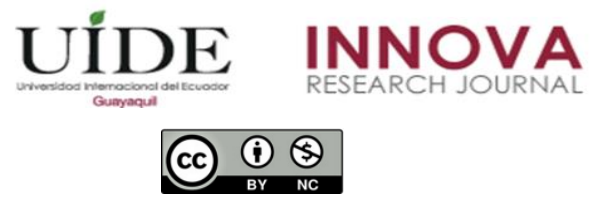

INNOVA Research Journal, ISSN 2477-9024

(Mayo-Agosto 2021). Vol. 6, No.2 pp.196-208

DOI: https://doi.org/10.33890/innova.v6.n2.2021.1621

URL: http://revistas.uide.edu.ec/index.php/innova/index

Correo: innova@uide.edu.ec

\title{
Análisis de los intereses moratorios en una empresa constructora
}

\section{Analysis of default interest in a construction company}

Victoria Gardi Melgarejo

Universidad Norbert Wiener, Perú

victoria.gardi@uwiener.edu.pe

https://orcid.org/0000-0001-6016-8719

Gerardo Arroyo Osorio

Universidad Norbert Wiener, Perú

gerardo.arroyo.osorio@gmail.com

https://orcid.org/0000-0001-6860-5848

Rosa Ysabel Moreno Rodríguez

Universidad Norbert Wiener, Perú

rosa.moreno@uwiener.edu.pe

(iD https://orcid.org/0000-0002-8357-4514

Recepción: 03/12/2020 | Aceptación: 03/03/2021 | Publicación: 10/05/2021

Cómo citar (APA, séptima edición):

Gardi Melgarejo, V., Arroyo Osorio, G., y Moreno Rodríguez, R. Y. (2021). Análisis de los intereses moratorios en una empresa constructora. Innova Research Journal, 6(2), 196-208.

https://doi.org/10.33890/innova.v6.n2.2021.1621

\section{Resumen}

El estudio se realizó con el objetivo de estudiar los elementos que inciden en el pago de los intereses moratorios, por lo cual es transcendental que las áreas implicadas en la práctica administrativa y financiera, posean el conocimiento contable y tributario. La investigación se desarrolla bajo el enfoque cualitativo, se hizo la recopilación de datos a través de las entrevistas a 5 trabajadores, con la respectiva guía de entrevista compuesta por seis preguntas alineadas a las sub categorías, permitiendo obtener una idea más amplia y más objetiva referente a los diferentes procesos que desarrolla la entidad, y mejorando factores claves que permiten que los efectos sean más precisos y concisos, luego se realizó la triangulación de los datos con el apoyo del software Atlas ti 8, en los resultados se pudo considerar que tanto los tributos, contraprestaciones con el estado y las sanciones administrativas inciden de forma directa en el pago de los intereses moratorios, en el sentido de que, cada impuesto que no era abonado genera intereses moratorios por día, así como había impuestos con multas por no ser abonadas, esto sucedía a menudo en la 
empresa constructora por la falta de preparación del colaborador del área de administración y finanzas, y la inadecuada comunicación entre las áreas implicadas, la importancia de no considerar el pago de estos impuestos en sus flujos.

Palabras claves: intereses moratorios; impuestos; capacitación; tributación; gradualidad; flujos y fraccionamiento.

\begin{abstract}
The study was carried out with the objective of studying the elements that affect the payment of default interest, which is why it is transcendental that the areas involved in administrative and financial practice have accounting and tax knowledge. The research is developed under a qualitative approach, the data was collected through the interviews with 5 workers, with the respective interview guide composed of six questions aligned to the sub categories, which allowed us to obtain a broader idea and more objective regarding the different processes that the entity develops, and improving key factors that allow the effects to be more precise and concise, then the triangulation of the data was carried out with the support of the Atlas ti 8 software, the results could be considered that both taxes, compensation with the state and administrative sanctions directly affect the payment of default interest, in the sense that each tax that was not paid generates default interest per day, as well as there were taxes with fines for not be paid, this often happened in the construction company due to the lack of preparation of the employee in the administration area and finances, and the inadequate communication between the areas involved, the importance of not considering the payment of these taxes in their flows.
\end{abstract}

Keywords: default interest; taxes; training; taxation; gradualism; flows and fractioning.

\title{
Introducción
}

Los intereses moratorios, en determinados países de Latinoamérica son valorados para poder aplicar el pago de intereses moratorios, concretamente en Chile la sentencia de la mayoría, el Tribunal Constitucional recoge exigencias de inaplicabilidad por inconstitucionalidad de ley, deducidos por un contribuyente, declarando que el inciso 3 del art. 53 del Código Tributario no logrará recibir aplicación en dos procesos de consulta definida, en los cuales el interesado es parte en contra de la atención de Impuestos Internos.

Todos los países poseen un aparato de aplicabilidad de intereses moratorios, en Argentina, que se entiende a este desembolso a sanear al acreedor no pagado, en término por la necesidad del manejo del dinero en el tiempo que duro el atraso, o por el costo que correspondería abonar en el mercado económico, para forjarse de una monto equivalente, en circunstancias de verse ajeno a esa suma, pero además indica que tiene que ser evaluado, el motivo del no reembolso, que éste a su vez genera dichos beneficios (Piccioni, 2016).

Sin embargo, las conveniencias de instaurar el código tributario en base a su aplicabilidad en ordenanzas de intereses moratorios, en los países Latinoamericanos se instituyen a un comisión que cada estado lo planteó y lo instituyó, caso contrario es en Bolivia, como estado busca que el contribuyente admita y acepte que incumplió, y eso determina sanciones e intereses por el incumplimiento del reembolso con su nuevo Código, que fija una tasa de interés gradual en la deuda tributaria, que es el $4 \%$ en los cuatro primeros años de deuda; luego, se acrecentará al $6 \%$ 
para los próximos tres años y el octavo año de deuda se determinara un interés del 10\% (Arce, 2016).

Uno de los elementos por lo que los intereses moratorios se acrecientan en nuestro país, es el tiempo que se toman para resolverlo, hace referencia cuando este posee de por medio una impugnación, entendiendo que en esa instancia actúan varios entes implicados para poder resolverlo. Cuando se refiere a montos, el impacto en estos intereses es considerable, por esa razón se entra en esa disputa (Gestion, 2015).

El artículo 33, del Texto Único Ordenado del código tributario alude que el interés moratorio nace por la falta de reembolso de un tributo en un tiempo determinado, este es remitido por Sunat, que reiteran la proporción de forma diaria o mensual. Así también, alude la forma de cálculo que logra ser realizado de modo diario o de modo mensual.

Constituye un fenómeno económico y no una imagen ideada el pago de intereses, es por ello por lo que logramos indicar que, el interés moratorio subsana los daños y perjuicios que la demora culpable o simulado lograse originar, además comprender que los lineamientos están fundados en normas y leyes legales como el Articulo 28 y 33 de Código Tributario que menciona las multas establecidas (Nuñez, 2014).

En la investigación de Maqson (2019) y Van (2018) aluden que los efectos en base a las tasas de intereses son en base al EBITDA, que posee una tendencia a variar por nuevas leyes, asimismo en base a los beneficios que se consiguen dar por las entidades de propiedad nacional y extrajera. Así mismos Ávila y Rodríguez (2016) concluyen que si bien es cierto los intereses moratorios es un reembolso que se realiza por norma, deberían adoptar incuestionables situaciones específicas para el instante en que sean definitivas, además que no correspondería ser tratados de la misma forma a los que tiene un compromiso por morosidad, teniendo como evidencia reiterado de dicho incumplimiento, por ende, las sanciones no deberían ser iguales para todos.

Braga (2015) menciona que las supervisiones y evaluaciones a los que tienen cargos directivos sean de manera permanente, ya que, si el tiempo es amplio, no sé daría una adecuada evaluación con respecto a sus funciones y obligaciones. Zagonel, Soares, y Favero (2015) detallan el grado de importancia de los impuestos, que se ejecuta para los diferentes gastos del estado, la inversión y las obligaciones, como gobierno.

En el caso peruano, De la Cruz (2018) concluyo que las empresas tienen un índice alto de morosidad, debido a la poca liquidez que no le admite cumplir con sus compromisos tributarios. Zumaeta y Chacaltana (2018) determinan, que el ente encargado Sunat pone muchos obstáculos para el contribuyente al punto de afectarlo, en las medidas de incautación sus cuentas, la exigencia del porcentaje de intereses pese a que este aún sigue en el tribunal fiscal desarrollándose.

Burgos y Gutiérrez (2016) concluyeron que la capacitación al personal debe ser una prioridad porque esto minimizaría el margen de error de cada área, se tiene claro que la empresa ante una fiscalización mostraría muchas faltas que se pueden evitar contando con personal idóneo. Gómez, Isla, y Mejía (2019) argumentan que las cifras porcentuales en base a los intereses 
moratorios, deben ser considerables de modo paulatino y estimados para que sean hechas de manera que no perjudique al deudor tributario.

Por lo tanto, el estudio se apoya en la teoría del Planeamiento y Planificación por Groppelli y Nikbakth (2012) quienes sostuvieron la importancia de contar con un plan financiero que consiga superar los inconvenientes inesperada en obra, además el área encargada debe tener personal idóneo y capacitada para poder cumplir con las obligaciones que requieren las entidades financieras y reducir el margen de error de los flujos proyectados que logren ejecutar el proyecto sin inconvenientes. Sunder (2005) y Ramírez (2008) refieren que la teoría de la contabilidad y control es importante porque el efecto de este proceso ayuda en la toma de decisiones posteriores, se debe controlar el progreso a detalle para lograr la rentabilidad de la compañía. Así mismo Cueva (2010), Schultz (1961) la teoría de las relaciones humanas permite comprender la importancia de la eficiencia y eficacia en el trabajo realizado por el personal destacado y que la empresa debe realizar retroalimentación a los cambios que se presentan en los procesos administrativos y financieros.

Gutiérrez (2016) y Pava y Rubiano (2017) conceptualizan los intereses moratorios, como una falta según lo determina la norma y ley que fue estipulada por cada estado, y que tiene como propósito cubrir determinados gastos de dicho estado, también conocer las discrepancias de los términos parecidos y conocer el porqué del esmero de estas sanciones contribuye a no realizarlas, ya que el problema no es solo en Perú, también son normas establecidas en otros países. Lo efectivo de esto es que en algunos países hay diferentes reuniones que se da por este tema, y Perú no es ajeno a ello, es evidente que los casos no son las mismas, pero sin embargo la norma es muy deficiente.

Es preciso señalar, que los intereses moratorios son causados por el incumplimiento en el pago de las obligaciones tributarias en una fecha establecida, y este tiene por finalidad reparar los daños y perjuicios que el retraso culpable o doloso puede causar. En tal sentido, las subcategorías son: a) Tributos, Contraprestaciones y aportes al sistema público b) Otros gastos de gestión, sanciones administrativas.

López (2017) señala que las obligaciones tributarias radican en el reembolso que toda persona natural según la ley está obligada a pagar por un periodo determinado, esto es manejado por el estado para cubrir los gastos de una Nación. Si el contribuyente incumple con ello incurre en una falta y esto determina una sanción tributaria.

Navarro (2019), afirma que la infracción tributaria se origina por la infracción de una norma que fue omitida por un contribuyente, es transcendental tener en cuenta que hoy existen normas, títulos y/o decretos legislativos que indican que, ante la falta de pagos tributarios, el interesado estaría cayendo en una trasgresión. Se entiendo también que el interés moratorio es el valor que pide la norma por el retraso del contribuyente ante la cancelación de los tributos, que se rige por un calendario expuesto por la SUNAT.

El estudio tiene como objetivo general: Analizar los intereses moratorios en una empresa constructora, y los objetivos específicos: a) Analizar en qué medida inciden los tributos, contraprestaciones y aportes al sistema público en el pago de los intereses moratorios en una 
empresa constructora; b) Analizar en qué medida inciden otros gastos de gestión, sanciones administrativas en el pago de los intereses moratorios en una empresa constructora.

\section{Metodología}

El enfoque de la investigación fue cualitativo, el mismo que consiste en comprender y ahondar toda la información establecida en la observación de diferentes comportamientos, explicaciones y réplicas abiertas para que sean detalladas y consecutivamente tenga una interpretación de resultados, es decir permite juntar numerosa información para luego ser agrupada, y en el estudio se manipule la información de tal forma que se pueda ver como apoya a la investigación y permita interpretar lo investigado (Galeano, 2018).

El método utilizado fue el estudio de caso, que permitió profundizar por medio de la documentación, que señala la realidad de lo que se está estudiando, el resultado permite entender el proceso del tema investigado, en efecto esta metodología consiente que la investigación tenga un mayor alcance, por ende, es más irrefutable en cuanto a la información recibida, (Simón, 2009), se aplicó el método analítico, porque permite que el análisis sea visto desde varios escenarios, descomponer la información que va ser analizada, visualizar mucho más material, delimitar el objetivo del estudio del problema de la entidad, (Hurtado y Toro, 2017).

Para la recopilación de los datos se aplicó la técnica de la entrevista a 5 colaboradores que ayudó a narrar los hechos en su contexto original y habitual de los participantes siendo estos: a) gerente de finanzas; b) contador general; c) asistente de finanzas; d) asistente de gestión de personas; e) el asistente de administración y finanzas.

El método de análisis que se utilizo fue la triangulación, que permitió afirmar los resultados y realizar la validación de evidencias cualitativos se empleó el programa Atlas ti 8, que ayudó a codificar los datos para realizar una investigación más profunda y dedicada a un objetivo común a través de la documentación, que debe contener una estructura pauteada de cumplimiento estricto para que esto cumpla su finalidad.

\section{Resultados}

En relación al proceso de triangulación a la subcategoría tributos, contraprestaciones y aportes al sistema público, se observar el nivel de importancia de los indicadores ya que algunos de ellos como Essalud, renta de quinta categoría y renta de cuarta categoría están complicados a una sanción administrativa por parte de Sunat, por lo tanto, el grado de importancia de esta triangulación. Por otro lado, la subcategoría otros gastos gestión, sanciones administrativas admitió ver las distintas carencias en el progreso administrativo por la poca preparación de los trabajadores que lo ejecutan, dichos vínculos son tal como se muestra en la figura 1. 


\section{Figura 1}

\section{Categorización de los intereses}

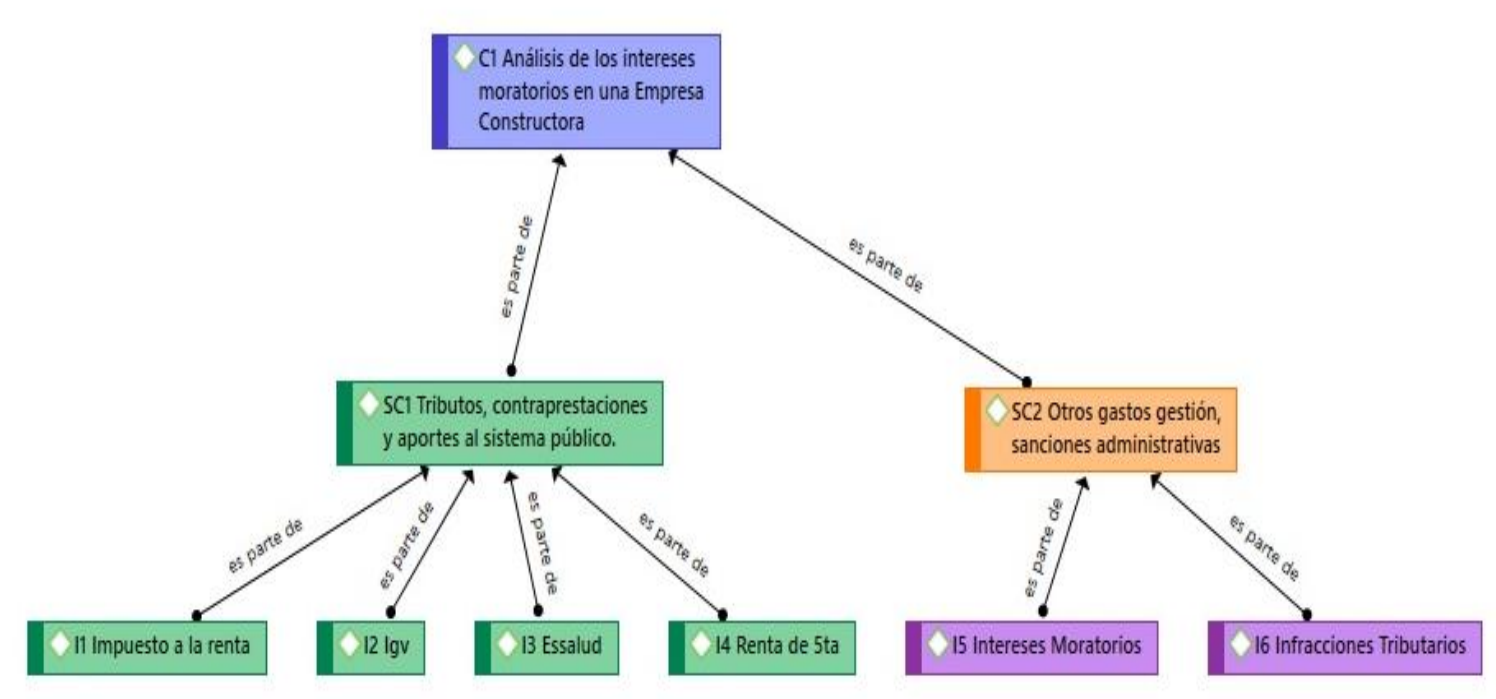

Existen varios factores que a la compañía le dificultaban poder ejecutar el cobro de las facturas, es por ello, que la empresa en ocasiones no podía hacer frente a sus obligaciones, como el pago de los impuestos, el personal desconocía la importancia de la cancelación de los impuestos, y tampoco tenía la información como por ejemplo del cronograma de Sunat, plazos de pagos, condiciones de contrato e inclusive conocimiento general de que impuestos sujetos a una sanción administrativa.

Los factores que inciden en el pago de intereses moratorios se deben a que la gerencia invertía en la compra de activos, mencionando que al realizarlo tendría más posibilidades en una licitación, porque cuando se postula, la empresa tiene que demostrar la capacidad en equipos, para que se pueda ejecutar la obra, también argumentaron que los pagos de las cuotas por leasing de los activos adquiridos eran prioridad para gerencia, esto repercutía en la liquidez de la empresa.

La inefectiva comunicación por parte del área administrativa, el área de finanzas, área contable, área legal y gerencia trae como consecuencia la ineficacia, la poca capacitación, no les permitía entender algunas situaciones importantes, la poca experiencia laboral provocaba errores, la constructora llegó a un punto de experimentar nuevas mecanismos de trabajo, porque ya no solo tercerizaba sino trabajaba de modo directo, empezaba a trabajar además con el estado y era por intermedio de fideicomisos, estos nuevos mecanismos financieros, mostraban procesos distintos de lo que la compañía ya venía desarrollando habitualmente,

Para efectuar un análisis a detalle se seleccionó un comparativo de la evolución de impuestos, en el cual se obtuvo en el 2015 un monto de S/488,296 soles, 2016 de S/492,259 soles, 
2017 S/2,621,697 soles y 2018 de 475,822 soles, este resultado permite observar que en relación a los años 2015, 2016 y 2018 no presenta un descenso de los impuestos, multas e intereses moratorios, todo lo contario por tres años consecutivos, en consecuencia los resultado permiten ultimar que durante ese tiempo no hubo medidas correctivas u opciones de posibles soluciones, como observamos en la tabla 1 .

\section{Tabla 1}

Análisis comparativo de Tributos

\section{Análisis de tributos 2015 - 2018}

\begin{tabular}{lcccc} 
Periodos & $\mathbf{2 0 1 5}$ & $\mathbf{2 0 1 6}$ & $\mathbf{2 0 1 7}$ & $\mathbf{2 0 1 8}$ \\
\hline Tributos por pagar & $488,296.00$ & $492,259.00$ & $2,621,697.00$ & $475,822.00$ \\
\hline
\end{tabular}

\section{Figura 2}

\section{Transición de tributos por pagar}

\section{Transición de Tributos}

\section{0}

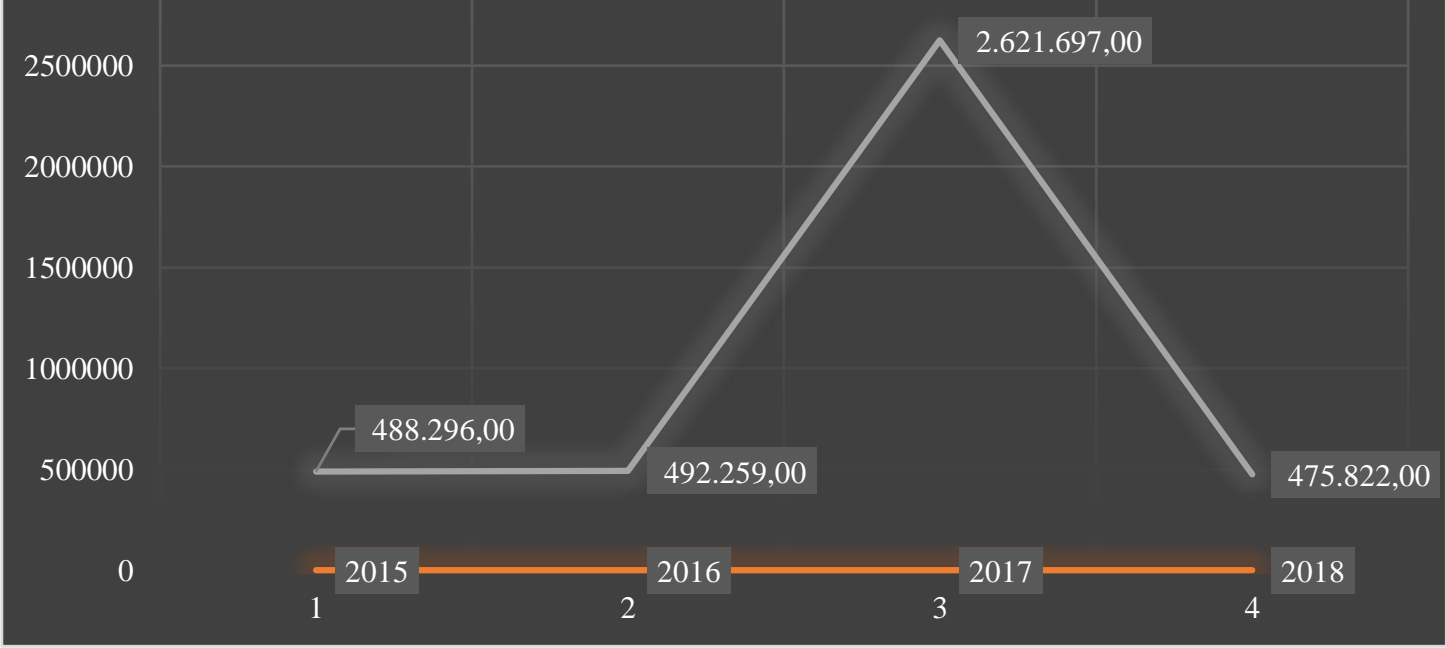

La compañía desconocía la importancia de la cancelación de los impuestos, más aún los efectos que este incumpliendo trae, como las deudas que estaban en cobranza coactiva, es por ello, que era necesario tener en cuenta la norma, que refiere los procesos que se mencionan en el Reglamento del procedimiento de cobranza coactiva de la Sunat que se acoge en la Resolución de Superintendencia N. ${ }^{\circ}$ 216-2004/SUNAT, teniendo en cuenta que el Código Tributario en el Titulo 
II detalle el Modo de Cobranza Coactiva, desde el artículo 114 hasta el artículo 121, destacando el artículo 117 que habla del procedimiento y el artículo 118 que se refiere a la medidas cautelares que la compañía en muchas ocasiones se ha encontrado, y la entidad se ha visto en la difícil situación que sus estados bancarias han sido retenidas por esta causa.

Las políticas que cobranza que maneja el estado eran prolongadas, otro factor que incidía era el tiempo que se demoraban en el área de ingeniería por parte de la empresa, ya que para poder valorizar se tenía que cumplir con los plazos que se habían proyectado, estos se veían perjudicados por la poca capacidad de abastecimiento de materiales en la provincia donde se ejecutaba el proyecto, porque retrasaba los plazos y la entidad se veía en la necesidad de llevar los materiales desde lima perjudicando en la facturación.

En el análisis del impuesto de Renta de tercera categoría, observamos, que en el año 2015 se refleja S/234,346 soles, en el 2016 S/230,100 soles, en el 2017 S/ 1,802,572 soles y el 2018 $\mathrm{S} / 281,572$ soles esto permite adelantar que la mayor parte de los intereses moratorios proceden de este gravamen, factor significativo para analizar medidas a tomar en cuenta y así poder neutralizar, el monto de S/ 2,548,865 soles, es considerable. Así mismo, vemos que el impuesto general a las ventas, refleja en el periodo 2015 un monto de S/ 132,500, en el 2016 S/135,700, en el 2017 S/ 575,000 Y $2018 \mathrm{~S} / 115,250$ de forma progresiva podemos determinar en base a estos periodos es los montos son grandes.

Verificamos que en Essalud en los cuatro periodos tenemos una suma de S/66,360 soles, por ser una compañía constructora estos costos varían por que la contratación de trabajadores es diferente de acuerdo a las labores que pueda haber durante el año.

La renta de quinta categoría, en los cuatro periodos la suma es de S/109,830 soles, en este caso según lo citado por la gerente de finanzas la compañía se enforzaba en cumplir con este impuesto, pero que en algunos meses no eran incluido en los flujos que pedía el área de finanzas por el desconocimiento e importancia de pagarlo. Como se muestra en la tabla 2.

\section{Tabla 2}

Comparativo de tributos por pagar 2015 al 2018

\begin{tabular}{llccccc}
\hline \multicolumn{1}{c}{ Cuentas } & Tributos & $\mathbf{2 0 1 5}$ & $\mathbf{2 0 1 6}$ & $\mathbf{2 0 1 7}$ & $\mathbf{2 0 1 8}$ & Totales \\
\hline \multirow{3}{*}{ Tributos } & Renta 3era & $234,346.00$ & $230,100.00$ & $1,802,847.00$ & $281,572.00$ & $2,548,865.00$ \\
por pagar & IGV & $132,500.00$ & $135,700.00$ & $575,000.00$ & $115,250.00$ & $958,450.00$ \\
& Renta 5ta & $12,200.00$ & $13,500.00$ & $75,630.00$ & $8,500.00$ & $109,830.00$ \\
Multas & Essalud & $12,000.00$ & $13,000.00$ & $35,860.00$ & $5,500.00$ & $66,360.00$ \\
& Renta 5ta & $52,500.00$ & $54,259.00$ & $75,860.00$ & $42,000.00$ & $224,619.00$ \\
& Essalud- & & & & & \\
Intereses & renta 5- Igv - & $44,750.00$ & $45,700.00$ & $56,500.00$ & $23,000.00$ & $169,950.00$ \\
moratorios & Renta 3era & & & & & \\
\hline
\end{tabular}


La falta de conocimiento por parte de las asistentes de finanzas y la asistente de gestión de personas, no ayuda a reducir las contingencias, en este caso al pago de intereses moratorios, se tiene que lograr, generando mayor conocimiento, innovación y constante capacitación para que el personal contribuya con los objetivos y metas empresariales según Mejía, Bravo y Montoya (2012).

\section{Figura 3}

Multas del periodo 2015 al 2018

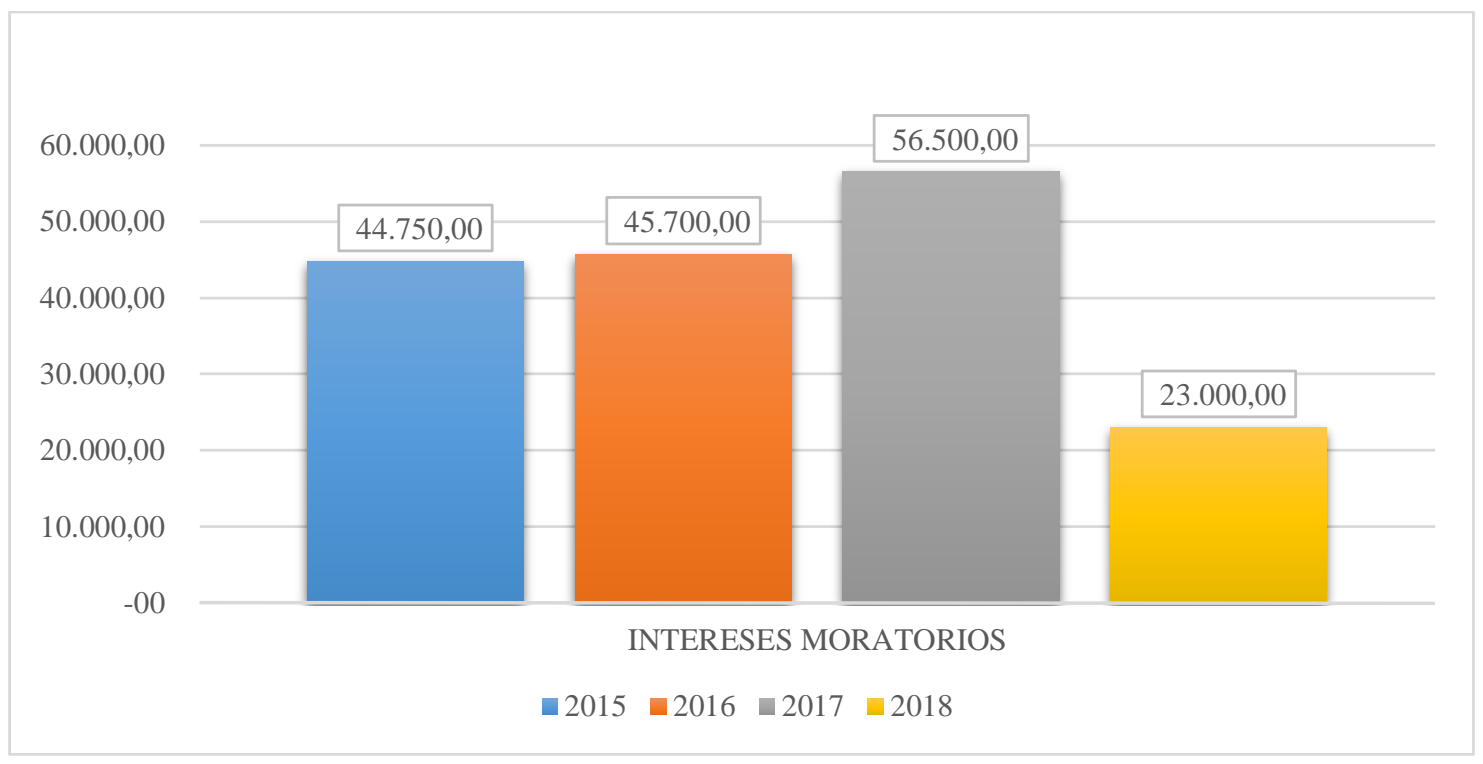

En referencia al análisis de los montos acumulados por multas, vemos que en renta de quinta categoría tenemos en el 2015 un monto de S/52,500 soles, 2016 un monto de $S / 54,259$ soles, 2017 un monto de $S / 75,860$ soles y 2018 un monto de S/42,000 soles, esto demuestra que pasa el tiempo y la compañía no ha bajado estos costos por las multas. Como observamos en la figura 5.

En intereses moratorios observamos en el año 2015 un monto de S/44,750 soles, 2016 un monto de S/45700 soles, 2017 un monto de S/56,500 y 2018 un monto de S/23,000 soles también observamos de manera creciente la cancelación de estos intereses refleja una reducción de ello. Teniendo un costo de S/394,569 soles por cuatro periodos, monto que la compañía paga en la actualidad, como se observa en la figura 4. 


\section{Figura 4}

Intereses moratorios del periodo 20215 al 2018

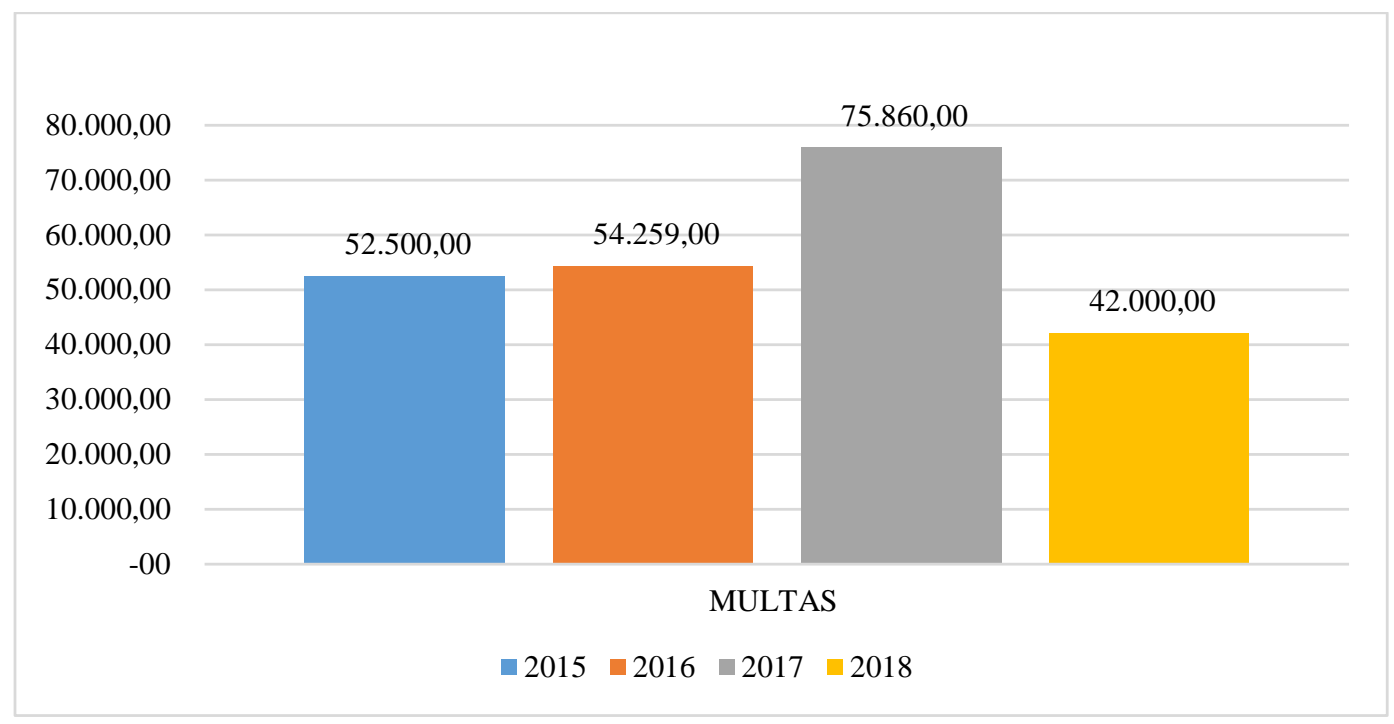

El impacto de los intereses moratorios de modo proporcional proviene en un $62 \%$ de la renta de tercera categoría, cabe indicar que, en el 2017, el mes de noviembre y diciembre la constructora facturó S/ 19,057,259 soles, la organización puede acogerse a formas graduales de pago que establece la Resolución de Superintendencia N.o 180-2012/SUNAT, la compañía al aplicarlas se hallaba en un resarcimiento inducida en cobranza aplicando el $60 \%$ afectando a la empresa, como se observa en la figura 5.

\section{Figura 5}

Representación porcentual de tributos

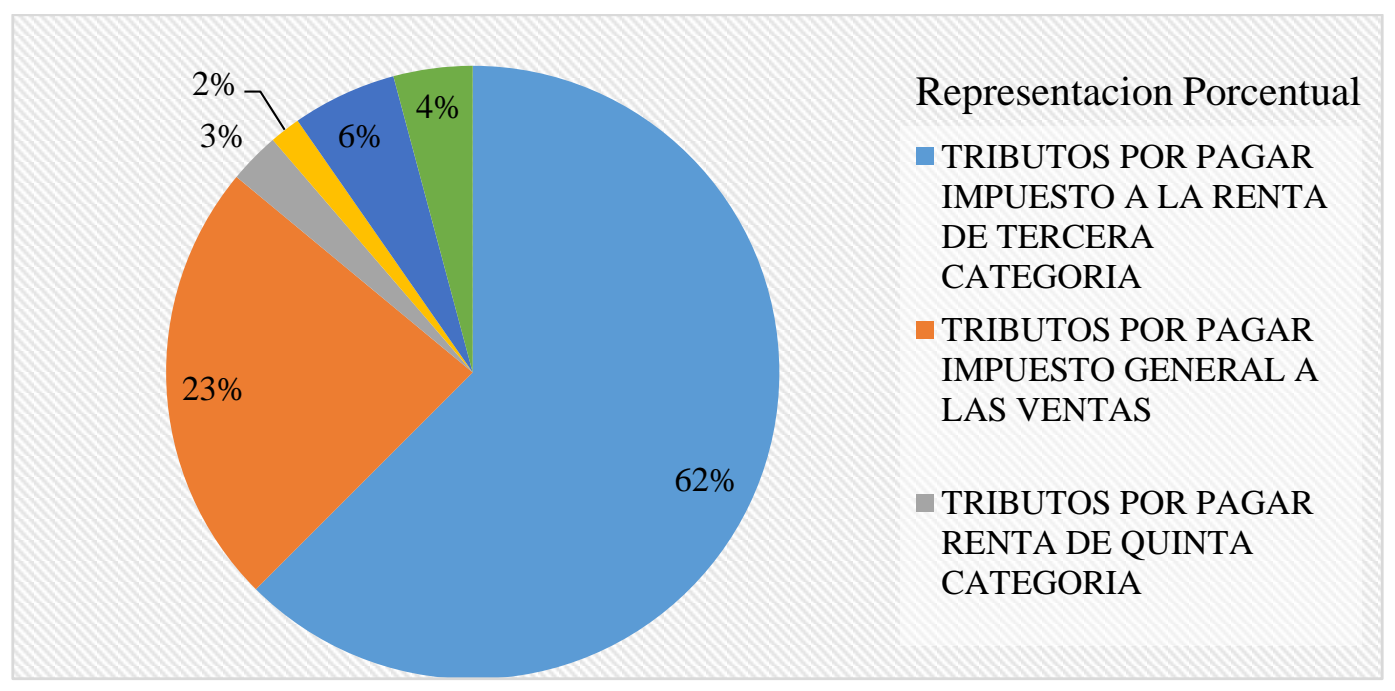




\section{Conclusiones}

Los elementos que inciden en el pago de intereses moratorios son la falta de pago de tributos, que en los periodos del 2015 hasta el 2018 reflejan costos significativos al igual que los costos por intereses moratorios y sus respectivas sanciones.

El personal delegado para la cancelación de tributos, carece de la preparación necesaria para entender la implicancia que conlleva no ejecutar estos pagos, tampoco tienen acceso a material necesario emitidos por la entidad, y las capacitaciones no son permanentes, en los temas contables y tributarios las normas cambian constantemente. Además, la gerencia no considera obligatorio dentro de sus flujos el pago de tributos, considerándolos obligaciones que carecen de importancia a pesar que sus contratos con diferentes obras lo requieren como exigencia para valorizar.

La responsabilidad que desboca en el pago de los intereses moratorios, proviene de la incompetencia de gerencia, que desconoce la importancia de todo el proceso administrativo por Sunat, por la pérdida de los fraccionamientos, por no abonar las retenciones, esto por falta de liquidez que se transforma en un círculo que tiene como resultado que la empresa no cuenta con el capital para hacer frente al problema.

\section{Referencias Bibliográficas}

Arce, L. (17 de Junio de 2016). La tasa de Interés de la deuda Tributaria. La tasa de Interés de la deuda Tributaria es de 10\% como tope, pág. 4. Obtenido de https://www.paginasiete.bo/economia/2016/6/17/tasa-interes-deuda-tributaria-como-tope99898.html\#!

Ávila, J., y Rodríguez, D. (2016). Condiciones especiales de pago e intereses moratorios. Herramienta de recaudo o instrumento nocivo para el sistema tributario. Revista de Derecho Fiscal, 7-28.

Braga, A. (2015). Corto plazo vs Largo plazo y la decisión de las estrategias en los incentivos. (Tesis de Licenciatura) Sau Paulo: Universidad de Sau Paulo.

Burgos, A., y Gutiérrez, D. (2016). La auditoría preventiva y su impacto en el riesgo tributario en la empresa Inversiones Padre Eterno EIRL año 2012 [Tesis de Licenciatura, Universidad Privada Antenor Orrego]. http://repositorio.upao.edu.pe/handle/upaorep/122

Cueva, A. (2010). Gestión del Talento Humano y del Conocimiento. En G. d. Talento. Bogotá, Colombia: Ecoe Ediciones.

De La Cruz, J. (2011). La política de crédito en la empresa. Empresa Actual, 5. https://www.empresaactual.com/la-politica-de-credito-en-la-empresa/

Galeano, M. (2018). Diseño de Proyectos de la Investigación Cualitativa. Medellín, Medellín, Colombia: Fondo Editorial. https://books.google.es/books?hl=es\&lr=\&id=Xkb78OSRMI8C\&oi=fnd\&pg=PA11\&dq 
$=$ enfoque + cualitativo \&ots $=$ zrLA8PUMvR\&sig=2lgY3uTRRld0y4nGCBlFenYtnGg\#v=o nepage $\& \mathrm{q}=$ enfoque $\% 20$ cualitativo $\& \mathrm{f}=$ false

Gestión. (30 de Septiembre de 2015). Intereses Moratorios. (C. Valencia, Ed.) Intereses Moratorios Vinculados al pago de impuesto a Sunat, pág. 1. https://gestion.pe/economia/intereses-moratorios-vinculados-pago-impuestos-sunat101185-noticia/?ref=gesr

Gómez, H., Isla, S., y Mejía, G. (2019). Apuntes sobre la Graduación de Sanciones por Infracciones a las Normas de Protección al consumidor. Revistas UPC, 1-13.

Groppelli, N. y. (2002). Planeamiento Financiero: Su importancia y su Contribución para la Gestión de las Empresas Cooperativas. Visión de Futuro, 3, 11. https://www.redalyc.org/pdf/3579/357935462004.pdf

Gutiérrez, A. (2016). Implicancia Financiera de la actualización de intereses por el pago extemporáneo de la Deuda Tributaria. Quipucamayoc, 10.

Hurtado, L., y Toro, G. (15 de Diciembre de 2017). El uso de los métodos deductivo e inductivo. Pontificia Universidad Católica Javeriana, 23. https://doi.org/10.11144/Javeriana.cc1846.umdi

López, P. (2017). Población muestra y muestreo. Scielo, 6. http://www.scielo.org.bo/pdf/rpc/v09n08/v09n08a12.pdf

Maqson, A. (2019). Optimización del proceso de selección de contratistas y evaluación de ofertas en la industria de la construcción: perspectiva del cliente. Revista de la Construcción, 114. https://scielo.conicyt.cl/pdf/rconst/v18n3/0718-915X-rconst-18-03-00445.pdf

Masbernat muñoz, P. (2013). Sentencia del tribunal constitucional de chile, rol $\mathrm{n}^{\circ}$ 1951(1952)2011-ina (acumulados)*, de 13 de septiembre de 2012, relativa a la inaplicabilidad de normas reguladoras del cobro de intereses por mora en el pago de tributos (ministro redactor sr. Enrique navarro; ministro redactor del voto disidente Sr. Gonzalo García). Alcances dogmáticos ¿qué hay de relevante? Acerca de instituciones, principios y reglas. Revista de derecho (Coquimbo), 20(2), 439-456. https://dx.doi.org/10.4067/S071897532013000200017

Navarro, J. (2019). ¿Que son las infracciones Tributarias? Grupo Verona, 1-2. Obtenido de https:/grupoverona.pe/que-son-las-infracciones-tributarias/

Nuñez, S. (2014). ¿Cuándo pagar intereses moratorios? Lima. Lima: Pontificia Universidad Católica del Perú.

Pava, M., y Rubiano, V. (2017). Impacto generado para las Pymes con el cálculo de intereses de mora según reforma Tributaria Ley 1607 año 2012. \{ Tesis de grado, Universidad Libre, Colombia]. http://hdl.handle.net/10901/10754

Piccioni, I. (2016). Infracciones y Sanciones Tributarias. [Tesis de maestría, Universidad Técnica de Machala, Ecuador]. http://repositorio.utmachala.edu.ec/handle/48000/6570

Simon, H. (2009). El estudio del caso: Teoría y práctica. London, Estados Unidos: Ediciones Morata S.L.

Sunder, S. (2005). Teoría de la Contabilidad y el Control. Bogotá: Internacional Thomson Publishing.

Zagonel, T., Soares, P., y Favero, D. (2015). Fiscalidad, gobierno corporativo y política de dividendos en Brasil. Scielo, 1-20. http://www.scielo.br/pdf/rmj/v53n3/2531-0488-RMJ53-3-0304.pdf 
Zumaeta, L., y Chacaltana, D. (2018). Problemas de Constitucionalidad a raíz de la entrada en vigencia de la Ley 30230 ¿Los intereses moratorios deben ser asumidos por el contribuyente cuando la demora en resolver es imputable al tribunal Fiscal? [Tesis de grado, Universidad Peruana de Ciencias Aplicadas, Perú]. https://oatd.org/oatd/record?record=handle\%5C\%3A10757\%5C\%2F623685 